\title{
Transient third nerve palsy after electrometallicthrombosis of carotid cavernous fistulae
}

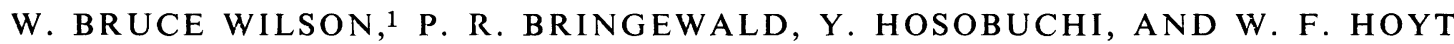 \\ From the Neuro-Ophthalmology Unit, Departments of Neurology, Neurological Surgery, and \\ Ophthalmology, University of California, San Francisco, California, USA
}

SYNOPSIS Three patients had oculomotor nerve palsy as a complication of the treatment of carotid cavernous fistulae by electrometallicthrombosis of the cavernous sinus. In two, third nerve function returned without misdirection in two months or less. One was left with a partial third nerve palsy, also without misdirection.

From July 1969 to July 1973, 15 cases of carotid cavernous fistula were treated by the electrometallicthrombotic technique by one of the authors (Y.H.). The surgical procedure involves exposure of the cavernous sinus through a frontotemporal carniotomy and transdural threading of fine copper or copper alloy wires into the lumen of the sinus. A low amperage direct current of electricity is applied to the cut ends of the wires to aid in the thrombosis of the sinus (Hosobuchi, 1975). This paper describes three cases of intracavernous ischaemic oculomotor neuropathy after transcranial electrometallicthrombosis of the cavernous sinus for carotid-cavernous fistulae. The cause of the oculomotor palsy and its normal pattern of recovery will be discussed in terms of the effects that surgery has on the blood supply to the neural structures traversing the cavernous sinus.

\section{CASE 1}

A 35 year old woman was referred to the University of California Neurosurgical Service two weeks after the onset of a bruit in her right ear. Mild proptosis and slight visual blurring of the right eye had been present for ten days.

At the time of admission the examination of the left eye was normal in all respects. The right eye was

\footnotetext{
${ }^{1}$ Address for reprint requests: Dr Bruce Wilson, Division of Ophthalmology, Denver General Hospital, Denver. Colorado 80204, USA.

(Accepted 9 January 1976.)
}

exotropic and had $7 \mathrm{~mm}$ of proptosis. There was marked swelling of the lids and conjunctiva; the veins were dilated and tortuous in the conjunctiva and retina. Abduction was full, but elevation, depression, and adduction were all reduced by approximately 25 per cent. The right upper lid was slightly ptotic. The right pupil was $5 \mathrm{~mm}$ in diameter and reacted poorly to light and accommodation. Other abnormal findings of the right eye were: (1) a visual acuity of $20 / 50,(2)$ a depression of the field of vision throughout, and (3) an intraocular pressure of $28 \mathrm{~mm} \mathrm{Hg}$ (normal value being $10-20 \mathrm{mmHg}$ ).

Selective carotid arteriography with subtraction and magnification showed the right internal carotid artery immediately opacifying the entire cavernous sinus. No external carotid or vertebral artery contribution was seen. Blood from the fistula drained anteriorly into a markedly dilated right superior ophthalmic vein and posteriorly into the right supcrior petrosal sinus.

Four weeks after the onset of symptoms the cavernous sinus was approached directly through a right frontotemporal craniotomy. The dura mater of the sinus appeared normal. Fine copper and copper alloy wires were threaded into the sinus through three routes: Parkinson's triangle posteriorly (see Discussion), the pterygoid plexus inferiorly, and the sphenoparietal sinus anteriorly. The wires were cut flush with the wall of the sinus. No direct current was used.

Although eye movements had remained stable before the operation, within four hours after the operation, adduction, elevation, and depression were all reduced to $50 \%$ of normal in the right eye. The amount of ptosis had increased and the pupil was fixed at $7 \mathrm{~mm}$. Twelve hours after the operation the 
right third nerve palsy was total. The right eye gradually lost all abduction during the first postoperative week.

Four weeks after surgery partial return of right third nerve function was first seen. With the exception of the pupil, which was $4 \mathrm{~mm}$ in diameter and fixed, there was complete return of function without misdirection by the end of the eighth postoperative week. On down gaze the previously paralysed levator palpebrae inhibited fully. The fourth and sixth nerves showed full function by the end of the sixth postoperative week. Proptosis began to subside within two weeks after surgery and gradually cleared over a six week period.

COMMENT This case demonstrates a pattern of total ophthalmoplegia which cleared completely after cavernous sinus electrometallicthrombosis. The third nerve showed no misdirection.
CASE 2

A 25 year old woman spontaneously developed proptosis in her right eye. Nine months later she was seen by the authors. Pulsatile proptosis measured $10 \mathrm{~mm}$. At the time of admission, the examination of the left eye was normal.

The right upper eyelid was markedly swollen but chemosis was mild. There were dilated veins in the lids, conjunctiva, and retina of the right eye. The eye had full movement, there was no ptosis, and the right pupil was normal. The visual field was full, visual acuity was $20 / 20$, and the intraocular pressure was normal. A loud bruit was heard over the entire head but it was loudest over the right eye.

Carotid arteriography showed an angiomatous carotid cavernous fistula on the right filling from the right internal carotid artery (Fig. 1), the right external carotid artery (Fig. 2), as well as the left internal carotid artery through intercavernous carotid

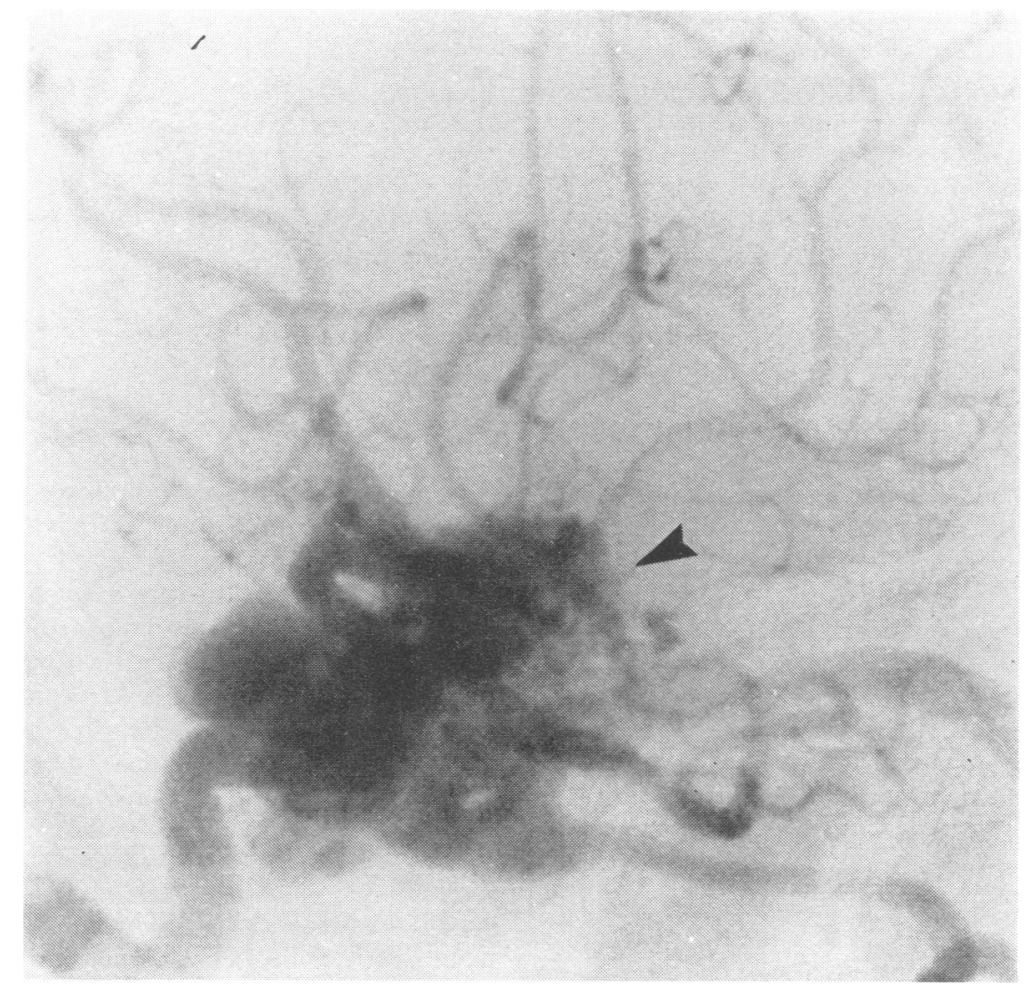

FIG. 1 Case 2. The right internal carotid arteriogram shows a rather unusual carotid cavernous fistula from a dural angioma (arrow). 


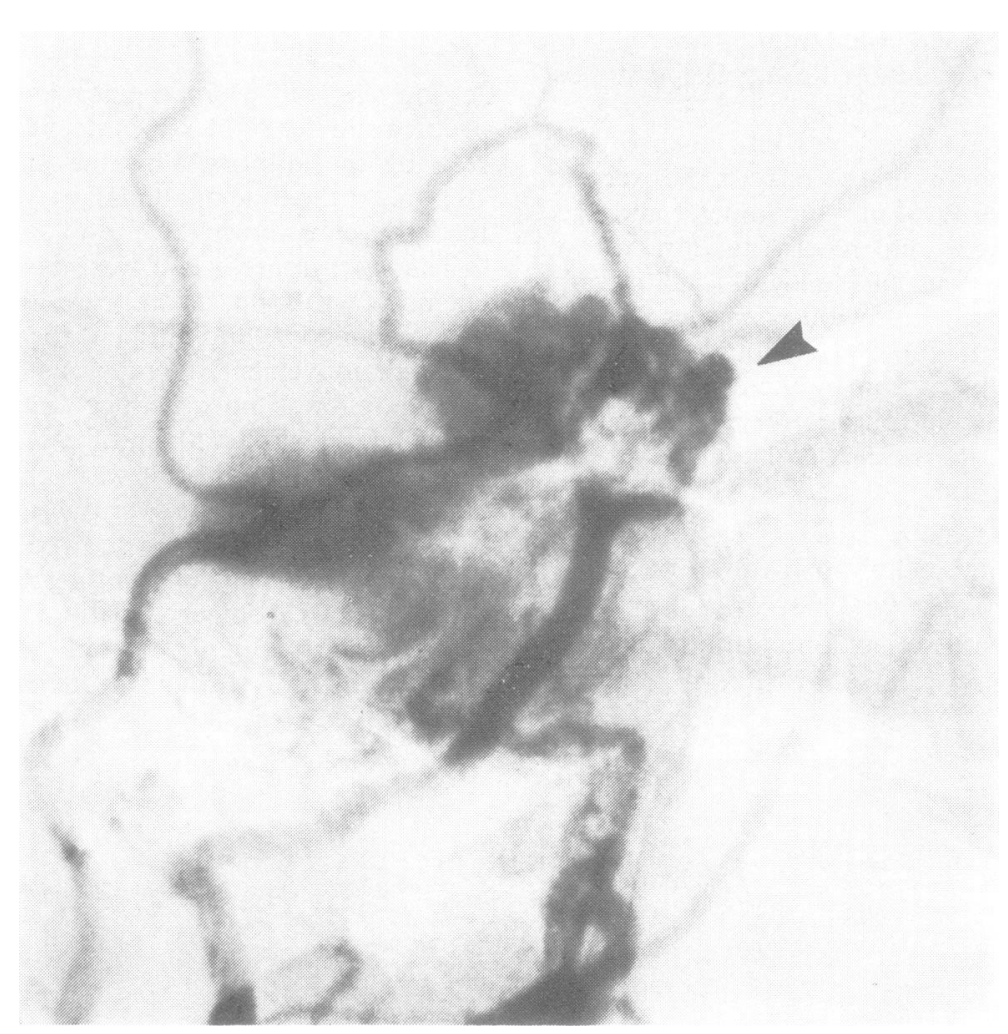

FIG. 2 Case 2. The right external carotid arteriogram shows the filling of the fistula through the same dural angioma (arrow).

branches (Fig. 3). The sinus primarily drained anteriorly through the right superior ophthalmic vein and, to a minor degree, through the right superior petrosal sinus.

The fistula was occluded by electrometallicthrombosis of the right cavernous sinus (Fig. 4). As in case 1, fine copper and copper alloy wires were placed into the sinus through Parkinson's triangle, the pterygoid plexus, and the sphenoparietal sinus. No current was applied.

In the recovery room immediately after surgery, the patient had a total right third nerve paresis which included the pupil. There was no palsy of the fourth and sixth cranial nerves. The third nerve palsy improved slowly and without misdirection over the next six weeks. Partial function was first seen at three weeks. Vision remained $20 / 20$ in the right eye and the proptosis gradually cleared over the six weeks after surgery. For several months after the operation the patient complained bitterly of right periorbital pain which later gradually decreased. Three years postoperatively she still has mild right third nerve palsy manifested by mild lack of elevation, depression, and adduction of the right eye with slight ptosis. The right pupil is also slightly larger than the left. She reports occasional blurring of right side vision though there seems to be no diplopia. There is no misdirection of the right third nerve.

COMMENT A total third nerve palsy was produced by electrometallicthrombosis of the cavernous sinus. The palsy cleared almost completely without misdirection. There was no postoperative palsy of the fourth and sixth cranial nerves. 


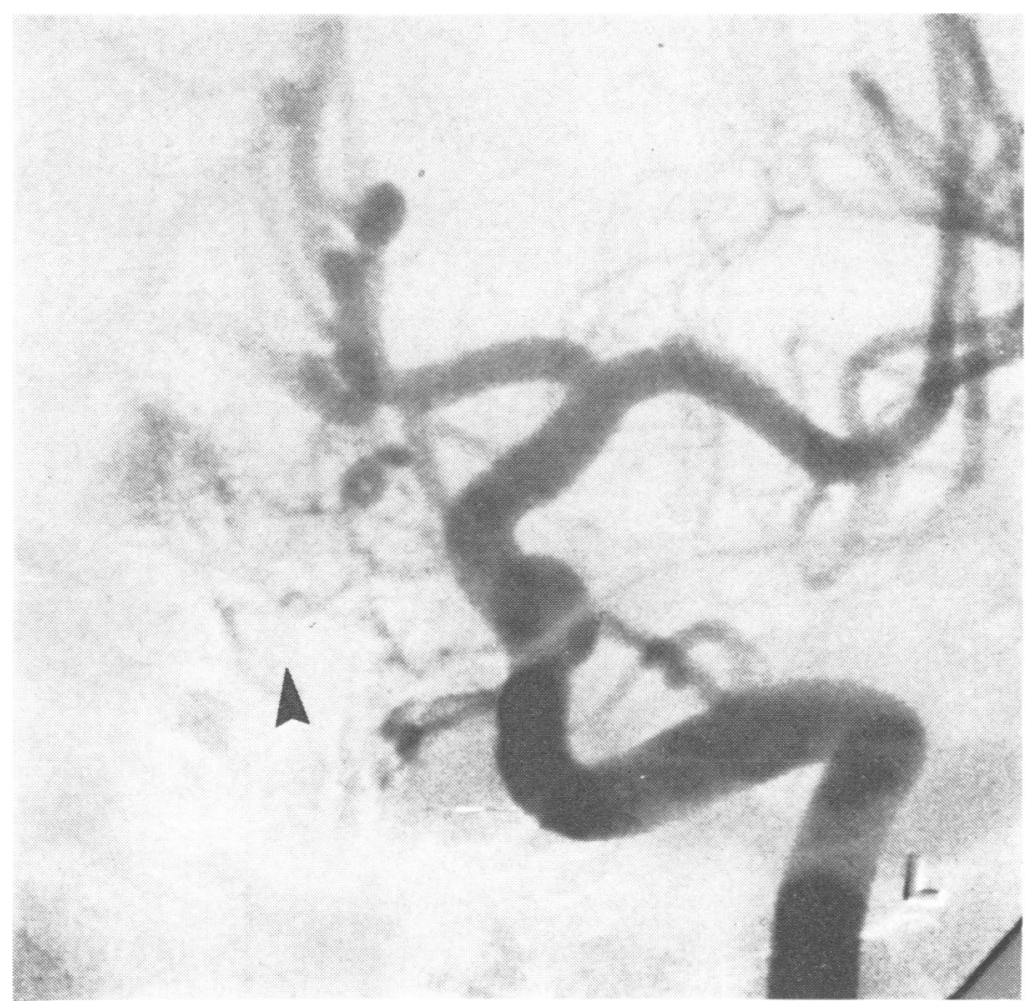

FIG. 3 Case 2. The left internal carotid arteriogram shows the filling of the fistula through the anastomotic branches (arrow) of the cavernous part of the carotid artery.

CASE 3

As the result of a basal skull fracture, a 17 year old man had bilateral sixth nerve palsy and a left seventh nerve palsy. Five weeks after the accident he noticed the onset of proptosis of the left eye, a bruit on the left side of his head, and pain in the left periorbital area.

Seven weeks after the injury he was admitted to the University of California Neurosurgical Service with $3 \mathrm{~mm}$ of proptosis of the left eye. Examination of the right eye was normal. Except for the loss of abduction, ocular movements of the left eye were full, there was no ptosis, and the left pupil reacted normally to light and accommodation. Additional positive findings of the left eye were: (1) visual acuity of 20/60, (2) general depression of the field of vision, (3) marked oedema of the optic disc, and (4) dilated retinal veins. Intermittently, he exhibited dysphasia accompanied by right hemiparesis. Both of these findings could be made worse if the left common carotid artery was compressed during an attack.

Four vessel angiography was done. Selective views of the left internal carotid artery showed the contrast material opacifying the entire cavernous sinus and the left superior ophthalmic vein, while the left hemispheric arteries filled poorly and with some delay. Also, there was cross flow to the fistula from the right internal carotid artery and from the basilar artery.

A left frontotemporal craniotomy was done and copper wires were threaded transdurally into the anterior and posterior parts of the cavernous sinus. Direct current was used to occlude the fistula (total $800 \mu \mathrm{A}$ for five minutes).

A left third nerve palsy that spared the pupil was observed 12 hours after surgery. Postoperative 


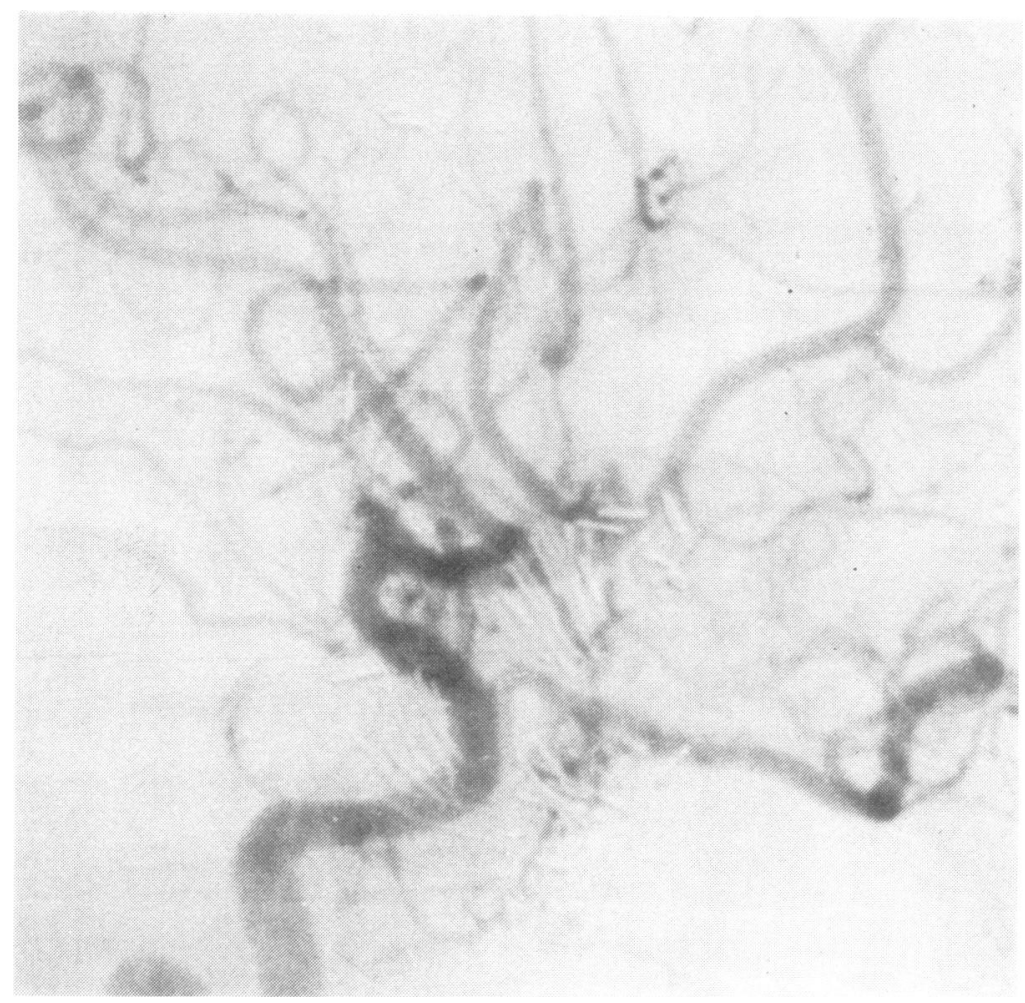

FIG. 4 Case 2. The postoperative right internal carotid arteriogram show's the complete obliteration of the angioma and the fistula.

paresis included the left fourth cranial nerve. The left sixth nerve was paretic before the operation.

Left third and fourth nerve function returned partially by the third week after surgery and completely returned by the eighth week after surgery. There was no misdirection. The traumatic sixth and seventh cranial nerve palsies remained. The proptosis slowly cleared by the sixth week after surgery. Angiograms done at four days and eight months after operation showed no evidence of a fistula and there was restoration of normal circulation through the left internal carotid artery. The patient has had no further attacks of dysphasia or hemiparesis.

COMMENT This case demonstrates complete ophthalmoplegia except for pupil sparing. The palsy cleared completely without misdirection.

\section{DISCUSSION}

SURGICAL ANATOMY AND SURGICAL PROCEDURE The aim of electrometallicthrombosis of the cavernous sinus in carotid-cavernous fistulae is to obliterate the shunt by occluding the venous spaces of the sinus with thrombus. A frontotemporal craniotomy is performed to expose the cavernous sinus. Then copper or copper alloy wires of $0.05-0.08 \mathrm{~mm}$ (2-3 thousandth inch) diameter are threaded transdurally into the sinus through a 30 gauge needle. Sometimes larger diameter copper wires may be used. The surgeon continues to add wires by multiple puncture until complete thrombosis of the sinus occurs.

If the sinus drains posteriorly, the wires are threaded into the sinus through the posterior part 
of Parkinson's triangle (Parkinson, 1965). The triangle is bounded superiorly by the third and fourth cranial nerves, inferiorly by the sixth and ophthalmic division of the fifth cranial nerves, and posteriorly by the slope of the dorsum sellae and clivus.

There are three major sources of blood supply to the intracavernous portion of the third nerve. One source arises from the carotid artery within the triangle as shown by Asbury et al. (1970) who showed that blood is also supplied to the intracavernous portion of the third nerve from the posterior part of the circle of Willis and accompanies it across the subarachnoid space. A third origin of blood comes from the ophthalmic artery collaterals intraorbitally.

If the cavernous sinus drains inferiorly, the wires are threaded down into the pterygoid plexus. If the cavernous sinus drains anteriorly, the wires are introduced into it through the sphenoparietal sinus.

The copper and copper alloy wires have properties of softness, spring, and thickness closely equivalent to human hair. Because of these properties trauma to intracavernous neural structures is unlikely. Once the copper comes into contact with the blood in the sinus it begins to dissociate into cuprous and cupric ions. These ions attract the negatively charged formed elements in the blood such as red blood cells, white blood cells, platelets, and fibrinogen. Thus a thrombus is started. A small anodal current may be applied to the cut ends of the wires to enhance the dissociation of the copper, thereby promoting the speed of thrombus formation.

Intraoperative angiography is used to determine when thrombosis of the cavernous sinus is adequate to achieve the surgical objective. At the end of the surgical procedure, the wires are left intact. A detailed report of this procedure has been published (Hosobuchi, 1975).

POSTOPERATIVE OCULOMOTOR PALSY AND ITS RECOVERY Oculomotor palsy occurred after electrometallicthrombosis surgery in the three patients presented in this paper. Twelve other patients were operated on in the same manner as the three presented but each of those had a complete oculomotor palsy before surgery and were therefore excluded from the study.

The postoperative oculomotor palsy was transient with partial return of function within four weeks of surgery and complete return of normal function within eight weeks of surgery in two cases, partial in one. The oculomotor palsy was initially complete in two patients and spared the pupil in one. The palsy was immediate in one and evolved in the first 12 hours after surgery in two. Palsy of other cranial nerves accompanied the oculomotor palsy, the fourth nerve in two, the sixth in one. In one patient the oculomotor palsy was accompanied with severe periorbital pain simulating Tolosa-Hunt syndrome (Tolosa, 1954; Hunt, 1961).

MECHANISM OF OCULOMOTOR PALSY We have considered several possible mechanisms in regard to the oculomotor palsy. One possibility is mechanical trauma. Four points argue against this: (1) in two patients the palsy was delayed; (2) the wires are soft and pliable; (3) neither the oculomotor nerve nor its blood supply occupies the area where wire was introduced; and (4) misdirection of oculomotor axons did not occur (Walsh and Hoyt, 1969).

We do not believe that the direct current caused the palsy: (1) it was not used in cases 1 and 2 ; (2) the amount of current employed is too little to cause nerve damage (Mullan et al., 1964); the copper wires in the cavernous sinus are in an electrolyte solution (blood), and the current density is limited to their surface; therefore, electrolytic damage of adjacent tissue is unlikely. (3) effects of direct current would be expected immediately, but in two patients the palsy was delayed.

We do not believe that a low-grade inflammation of the cavernous sinus caused the palsy. The Tolosa-Hunt syndrome, which is a painful ophthalmoplegia due to cavernous sinus inflammation (Tolosa, 1954; Hunt, 1961; Lakke, 1962), bears some resemblance to our cases. While prolonged direct contact with copper can cause inflammation in neural tissue (Dymond et al., 1970), rapidity of onset of palsy, in our patients, probably excludes this aetiology.

We believe that ischaemia in the intracavernous portion of the oculomotor nerve caused the palsy. The tempo of onset was similar to that of an ischaemic oculomotor palsy (Asbury et al., 1970; Weber and Daroff, 1970). Also the duration of palsy and its recovery without misdirection are 
fully consistent with this aetiology. Asbury et al. (1970) have suggested that diabetic ischaemia of oculomotor neuropathy results from partial occlusion of several nutrient vessels. In our cases ischaemia was probably secondary to thrombosis in the cavernous sinus and resulting stagnant anoxia of oculomotor axons.

Zülch (1964) has suggested that third nerve palsy in carotid cavernous fistulae occurs from stretching and occlusion of small arterial twigs to a nerve. Although our cases of carotid-cavernous fistulae developed palsy after thrombosis of the sinus, it may be that ischaemia of the nerve was produced by this mechanism.

Von $\operatorname{Rad}$ et al. (1971) in a clinical study of spontaneous aseptic cavernous sinus thrombosis found third nerve palsy in five of 10 cases. The pattern of onset and recovery resembled our cases.

From the surgical point of view, regardless of its aetiology, the oculomotor palsy complicating electrothrombosis of the cavernous sinus can probably be regarded as a temporary inconvenience.

The authors express their gratitude to Dr Kemp Clark, Dr Charles Israel, and Dr Stuart B. Black, Dallas, Texas, for their kind assistance in the follow-up of case 2.

\section{REFERENCES}

Asbury, A. K., Aldredge, H., Hershberg, R., and Fisher, C. M. (1970). Oculomotor palsy in diabetes mellitus: a clinico-pathological study. Brain, 93, 555-566.
Dymond, A. M., Kaechele, L. E., Jurist, J. M., and Crandall, P. H. (1970). Brain tissue reaction to some chronically implanted metals. Journal of Neurosurgery, 33, 574-580.

Hosobuchi, Y. (1975). Electrothrombosis of carotid cavernous fistula. Journal of Neurosurgery, 42, 76-85.

Hunt, W. E. (1961). Painful ophthalmoplegia. Its relation to indolent inflammation of the cavernous sinus. Neurology (Minneap.), 11, 56-62.

Lakke, J. P. (1962). Superior orbital fissure syndrome: report of a case caused by local pachymeningitis. Archives of Neurology (Chic.), 7, 289-300.

Mullan, S., Beckman, F., Vailati, G., Karasick, J., and Dobben, G. (1964). An experimental approach to the problem of cerebral aneurysm. Journal of Neurosurgery, 21, 838-845.

Parkinson, D. (1965). A surgical approach to the cavernous portion of the carotid artery. Journal of Neurosurgery, 23, 474-483.

Tolosa, E. (1954). Periarteritic lesion of the carotid siphon with clinical features of carotid infraclinoid aneurysm. Journal of Neurology, Neurosurgery, and Psychiatry, 17, 300-302.

Von Rad, M., Wolf, P., and Tornow, K. (1971). Die Blande Thrombose des Sinus Cavernosus. Deutsche Medizinische Wochenschift, 96, 457-464.

Walsh, F. B., and Hoyt, W. F. (1969). Clinical Neuroophthalmology, pp. 253-257. Williams and Wilkins: Baltimore.

Weber, R. B., and Daroff, R. B. (1970). Pathology of oculomotor nerve palsy in diabetics. Neurology (Minneap.), 20, 835-838.

Zülch, K. J. (1964). Neurologische diagnostik bei endokraniellen komplikationen von otorhinologischen erkrankugen. Archiv für Ohren-, Nasen- und Kehlkopfheilkunde, 183, 1-78. 